\title{
EVALUACION DE METODOS DE PROPAGACION ASEXUAL DEL NOPAL (Opuntia Ficus indica), Y RESPUESTA A DIFERENTES SUSTRATOS EN EL MUNICIPIO LOS SANTOS SANTANDER
}

\author{
EVALUATION OF CACTUS PROPAGATION METHODS (Opuntia \\ Ficus indica), AND RESPONSES TO DIFFERENT SUBSTRATES IN \\ THE MUNICIPALITY LOS SANTOS - SANTANDER
}

\section{RESUMEN:}

Este trabajo se realizó en la zona rural del municipio de Los Santos - Santander, realizando el siguiente procedimiento:

Se realizó el embolsado para la siembra de los cladodios con los distintos sustratos, previamente mezclados en el caso de los que contenían gallinaza compostada. En total se empacaron 120 bolsas de la siguiente manera: 30 bolsas de tierra caliche con gallinaza compostada, 30 con tierra fértil con gallinaza compostada, 30 con tierra caliche sin adición de gallinaza compostada y 30 con tierra fértil sin adición de gallinaza compostada.

Luego se procedió a la recolección de los cladodios de nopal, se realizó el debido tratamiento a estos con caldo bordelés y cal, para evitar que fuera atacada por insectos y hongos, se dejaron madurar durante doce días a la sombra para evitar deshidratación de los mismos. El mismo día de la siembra se hizo la división de los cladodios en dos y en cuatro partes, para evitar que se deshidrataran.

La primera hilada se sembró cladodios completos, la segunda hilada con cladodios divididos en dos partes y la tercera con cladodios divididos en cuatro partes. Este orden de ubicación de los cladodios se hizo de la misma manera para todos los sustratos. En total se hicieron doce (12) tratamientos.

Ya teniendo el montaje del proyecto listo, diariamente se le hizo observación de la evolución del mismo. Toda esta información se tabulo para realizar el análisis correspondiente al final del proyecto. Los resultados fueron analizados con distintos programas estadísticos.

\section{PALABRAS CLAVES: Cladodio, Embolsado, Nopal, Sustrato, Tratamiento, Vivero.}

\section{SUMMARY:}

This work was carried out in the rural area of the municipality of Los Santos - Santander, carrying out the following procedure.

The bagging was made for the sowing of the cladodes (seed) with the different substrates, previously mixed in the case of those containing composted chicken. In total 120 bags were packed as follows: 30 bags of caliche land without the addition of composted chicken coop and 30 with fertile soil without the addition of composted chicken coop.

Then it proceeded to the collection of seeds (cladodes) cactus, the proper treatment was done to these broth bordeles and lime, to prevent it was attacked by insects and fungi, allowed to mature for twelve days in the shade to avoid dehydration of the same. On the same day of planting, the cladodes were divided into two and four parts, to prevent dehydration.

DOI: https://doi.org/10.22490/26653176.2341 
The first course was planted complete cladodes, the second course with cladodes divided into two parts and the third with cladodes divided into four parts. This order of location of the cladodes was done in the same way for all substrates. In total, twelve (12) treatments were made.

Once the assembly of the project was ready, it was daily observed the evolution of the project. All this information is tabulated to perform the corresponding analysis at the end of the project. The results were analyzed with different statistical programs.

\section{KEYWORDS: Cladodes, Bagguing, Cactus, Substrate, Treatment, Nursery.}

\section{INTRODUCCION:}

En Colombia el cultivo de Nopal no tiene mayor importancia económica, desaprovechando las bondades que este tiene como alternativas agropecuarias en zonas semi desérticas, con terrenos áridos y secos. El Nopal es un cultivo ecológico ya que necesita poca agua para su sostenimiento, ayuda a incorporar partículas de materia orgánica que estén presentes en el ambiente, sirve como forraje para ganado bovino, caprino y ovino, tiene diversos usos para el ser humano como en la preparación de yogurt, cremas, champú, harinas, en preparación de jugos o como verdura. Otro de los usos importantes, es la utilización como cerca viva, para ayudar a contener especies que invaden terrenos ajenos como son el ganado caprino y bovino.

El objeto principal del presente trabajo es evidenciar la viabilidad vegetativa del cladodio del Nopal en distintos sustratos y los más importante, dividiéndolo para aprovecharlo al máximo sacando de un cladodio dos (2) o cuatro (4) unidades meristemáticas, evidenciando así el buen desarrollo y crecimiento de la planta que de estas crezcan.

La forma más común de propagar el cultivo de Nopal, es utilizando la propagación asexual mediante el cladodio. Este tipo de propagación, asemeja a la propagación por estaca, ya que cuando se habla de cladodio, se refiere a una parte del tallo o ramas de la planta de Nopal.

El presente experimento se realizó en el municipio de Los Santos - Santander, Vereda Paso Chico, Granja Las Nieves, localizada a una Altitud de 1201 MSNM, Longitud -73,146639 y Latitud 6,782806 .

\section{METODOLOGIA:}

La siembra de los cladodios de Nopal se hizo en vivero en bolsa, ya que se pudo controlar de una mejor manera factores importantes para la toma de información como lo son el sustrato a utilizar, formación radicular, aplicaciones hídricas y finalmente el trasplante.

Los sustratos con los que se trabajó como distintos testigos son: sustrato de tierra caliche sin gallinaza compostada, sustrato de tierra caliche con gallinaza compostada, sustrato de tierra fértil sin gallinaza compostada y sustrato de tierra fértil con gallinaza compostada. Esto con el objetivo de observar en cuál de estos se desarrolla mejor la parte radicular de las plántulas, más rápido y con mayor vigorosidad.

El método de propagación asexual que se utilizó fueron cladodios de nopal recolectados de plantas de la zona para garantizar la viabilidad de la misma, ya que estas están adaptadas al suelo árido y clima propios de la zona. Al hablar de cladodios, se asemeja al método de propagación por estaca, ya que el cladodio da formación al tallo y ramas del nopal. Para cada tipo de sustrato se sembró diez (10) métodos de propagación con cladodios (figura No. 1): cladodio completo, cladodio dividido en dos (2) partes y cladodio dividido en cuatro (4) partes. Para un total de ciento veinte (120) cladodios sembrados en la misma cantidad de bolsas. 
El tipo de diseño experimental con el cual se trabajó para este proyecto es el factorial 4x3, ya que este diseño es muy útil cuando se quiere investigar la forma cómo influyen los factores sobre una variable respuesta y en cada factor se considera solamente tres niveles. El término "experimento factorial" o "arreglo factorial" se refiere a la constitución de los tratamientos que se quieren comparar. Diseño de tratamientos es la selección de los factores a estudiar, sus niveles y la combinación de ellos. El diseño de tratamientos es independiente del diseño experimental que indica la manera en que los tratamientos se aleatorizan a las diferentes unidades experimentales y las formas de controlar la variabilidad natural de las mismas.

Figura No. 1. Ubicación de los tratamientos dentro del vivero

\begin{tabular}{|c|c|c|c|c|c|c|c|c|c|c|c|}
\hline \multicolumn{3}{|c|}{$\begin{array}{c}\text { CALICHE CON } \\
\text { GALLINAZA } \\
\end{array}$} & \multicolumn{3}{|c|}{$\begin{array}{l}\text { FERTIL CON } \\
\text { GALLINAZA } \\
\end{array}$} & \multicolumn{3}{|c|}{$\begin{array}{l}\text { CALICHE SIN } \\
\text { GALLINAZA }\end{array}$} & \multicolumn{3}{|c|}{$\begin{array}{l}\text { FERTIL SIN } \\
\text { GALLINAZA } \\
\end{array}$} \\
\hline $100 \%$ & $50 \%$ & $25 \%$ & $100 \%$ & $50 \%$ & $25 \%$ & $100 \%$ & $50 \%$ & $25 \%$ & $100 \%$ & $50 \%$ & $25 \%$ \\
\hline X & X & X & X & X & X & X & X & X & $x$ & X & $X$ \\
\hline$X$ & $X$ & $X$ & X & $X$ & $X$ & X & X & $X$ & $X$ & $X$ & X \\
\hline X & X & X & $X$ & $X$ & X & $X$ & $X$ & $X$ & $X$ & $X$ & $X$ \\
\hline X & $X$ & $X$ & $X$ & $X$ & $X$ & X & X & $X$ & X & $X$ & $X$ \\
\hline $\mathrm{X}$ & $X$ & $\mathrm{X}$ & $\mathrm{X}$ & $X$ & $X$ & $\mathrm{X}$ & $\mathrm{X}$ & X & $X$ & $\mathrm{X}$ & $X$ \\
\hline$X$ & $\mathrm{X}$ & $X$ & $X$ & $X$ & $X$ & $X$ & $\mathrm{X}$ & $X$ & X & $\mathrm{X}$ & $X$ \\
\hline X & $X$ & X & $X$ & $X$ & $X$ & X & X & X & X & X & $X$ \\
\hline X & X & $X$ & $X$ & $X$ & X & $X$ & $X$ & $X$ & $X$ & X & $X$ \\
\hline$X$ & $\mathrm{X}$ & $X$ & $X$ & X & $X$ & $X$ & X & $X$ & $\mathrm{X}$ & $X$ & $X$ \\
\hline$X$ & $X$ & $X$ & $X$ & $X$ & $X$ & $X$ & $X$ & $X$ & $X$ & $X$ & $X$ \\
\hline
\end{tabular}

En la figura No. 1 se puede evidenciar la ubicación de cada método de propagación por cladodio del nopal en los respectivos sustratos. Los cladodios completos son los nombrados (100\%), los divididos en dos partes son (50\%) y los divididos en cuatro partes son (25\%).

Preparación de sustratos y embolsado: Para la preparación de los distintos sustratos a manejar para el llenado de bolsas, se utilizó tierra propia de la zona de la Vereda Paso Chico del municipio de Los Santos - Santander. Los cuales son tierra caliche, esta tiene alto contenido de calcio; y tierra fértil la cual es bastante arcillosa. Con estos dos tipos de suelos, se trabajaron cuatro sustratos, los cuales fueron: Tierra fértil sin adición de gallinaza compostada, tierra fértil con adición de gallinaza compostada, tierra caliche sin adición de gallinaza compostada y tierra caliche con adición de gallinaza compostada.

Recolección de cladodios de nopal: Para la adquisición de cladodios, se recolecto de distintas plantas de nopal nativas en la zona. Se recolectaron de plantas que presentaban una buena madurez vegetativa, sanidad, vigorosidad y un tamaño del cladodio ideal para su propagación.

Tratamiento de cladodios de nopal: Luego de recolectar todos los cladodios, se procede a sumergirlos por un tiempo de quince (15) minutos en un balde con $10 \mathrm{~L}$ de agua, que contenía una solución de $30 \mathrm{~g}$ de caldo bordelés con $1 \mathrm{Kg}$ de cal. En el caso de los cladodios divididos en dos y cuatro partes, se hizo el debido fraccionamiento el mismo día en que se sembró para así evitar posible mortalidad de estos a causa de deshidratación, ataque fungoso o de insectos, y se le realizo el mismo tratamiento de desinfección.

Siembra: Los cladodios se sembraron respetando el mismo orden en todos los tipos de sustrato. La primera hilera se sembró el cladodio completo (100\%), la segunda hilera se sembró el cladodio dividido en dos partes (50\%) y la tercera hilera se sembró el cladodio dividido en cuatro partes (25\%).

Análisis estadístico: Se calcularon las frecuencias y proporciones de los cladodios que tuvieron formación vegetativa hasta los 116 días de seguimiento, para cada tratamiento y para cada tipo de cladodio, y se compararon con la prueba exacta de Fisher. Se realizó un análisis de supervivencia de Kaplan Meier que permitió calcular la mediana del tiempo de formación radicular de acuerdo con el

Carlos Hernán Mateus Arango, 94475450, Agronomía, UNAD, canan64@hotmail.com 
tipo de sustrato y el tamaño de las mismas y se compararon las curvas de formación radicular con la prueba de Log-Rank.

Se evaluó la normalidad de las variables área del cladodio y de la raíz y el número de retoños primarios y secundarios de cada cladodio, en cada tratamiento y se encontró que ninguna tuvo distribución normal. Por lo tanto, se realizó la prueba no paramétrica de Kruskal Wallis para comparar los sustratos y los tamaños de los cladodios.

\section{DISCUSIONES Y RESULTADOS:}

Tabla 1: Proporción de cladodios con formación radicular y tiempo, de acuerdo al sustrato y método del cladodio sembrado

\begin{tabular}{|l|c|c|c|c|}
\hline \multirow{2}{*}{ Tratamiento (n) } & \multirow{2}{*}{$\begin{array}{c}\text { Cladodios } \\
\text { germinados }\end{array}$} & \multicolumn{2}{c|}{ Tiempo de germinación (días)* } \\
\cline { 3 - 5 } & $9(90)$ & 27 & 27 & 33 \\
\hline Caliche con gallinaza 100\% (10) & $10(100)$ & 33 & 28 & 54 \\
\hline Caliche con gallinaza 50\% (10) & $10(100)$ & 37 & 37 & 48 \\
\hline Caliche con gallinaza 25\% (10) & $10(100)$ & 21 & 21 & 21 \\
\hline Fértil con gallinaza 100\% (10) & $10(100)$ & 30 & 30 & 42 \\
\hline Fértil con gallinaza 50\% (10) & $10(100)$ & 33 & 28 & 37 \\
\hline Fértil con gallinaza 25\% (10) & $10(100)$ & 27 & 25 & 28 \\
\hline Caliche sin gallinaza 100\% (10) & $7(70)$ & 48 & 48 & 48 \\
\hline Caliche sin gallinaza 50\% (10) & $7(70)$ & 80 & 80 & 80 \\
\hline Caliche sin gallinaza 25\% (10) & $10(100)$ & 25 & 25 & 25 \\
\hline Fértil sin gallinaza 100\% (10) & $10(100)$ & 33 & 30 & 42 \\
\hline Fértil sin gallinaza 50\% (10) & $10(100)$ & 42 & 30 & 42 \\
\hline Fértil sin gallinaza 25\% (10) & & & & \\
\hline
\end{tabular}

${ }^{£}$ Valor p de Prueba exacta de Fisher $=0.004$

*Valor p de prueba de Log-rank $<0.001$

Al analizar el tiempo de formación radicular por sustrato, teniendo en cuenta el tamaño del cladodio, se pudo observar que el grupo que se desarrolló más rápido fue el de los cladodios completos sembrados en sustrato fértil con gallinaza compostada. Por el contrario, el que germinó más lento fue el de los cladodios cortados en cuartos y sembradas en caliche sin gallinaza compostada. Igualmente, en este sustrato fue donde se observó la mayor mortalidad de cladodios, como se observa en la tabla 1.

Tabla 2: Proporción de cladodios enraizados y tiempo de formación vegetativa de acuerdo a su método

\begin{tabular}{|l|c|c|c|c|c|}
\hline \multirow{2}{*}{ Tratamiento (n) } & \multirow{2}{*}{$\begin{array}{c}\text { Cladodios } \\
\text { enraizados }^{\mathfrak{\varepsilon}}\end{array}$} & \multicolumn{2}{|c|}{$\begin{array}{c}\text { Tiempo de formación } \\
\text { vegetativa (días) }\end{array}$} & \multirow{2}{*}{ Valor p* } \\
\cline { 3 - 5 } & & Mediana & \multicolumn{2}{|c|}{ IQR } & \\
\cline { 2 - 5 } & & 25 & 25 & 27 & \multirow{2}{*}{$<0.001$} \\
\cline { 1 - 4 } Cladodio 100\% (40) & $39(97.5)$ & 37 & 30 & 48 & \\
\hline Cladodio 50\% (40) & $37(92.5)$ & 42 & 33 & 48 & \\
\hline
\end{tabular}

${ }^{\mathrm{E}}$ Valor $\mathrm{p}$ de Prueba exacta de Fisher $=0.697$

*Prueba Log-rank

Al calcular el tiempo de formación radicular de acuerdo con el método del cladodio, es evidente que sembrarlo al método completo (100\%) favorece la formación temprana de la planta, mientras que los cladodios divididos en cuatro partes (25\%) se forman más lentamente, como se puede observar en la tabla 2

Tabla 3: Proporción de cladodios enraizados y tiempo de formación radicular de acuerdo con el tipo de sustrato 


\begin{tabular}{|l|c|c|c|c|c|}
\hline \multirow{2}{*}{\multicolumn{1}{|c|}{ Tratamiento (n) }} & \multirow{2}{*}{$\begin{array}{c}\text { Cladodios } \\
\text { enraizados }^{\mathfrak{t}}\end{array}$} & \multicolumn{2}{c|}{$\begin{array}{c}\text { Tiempo de formación radicular } \\
\text { (días) }\end{array}$} & \multirow{2}{*}{ Valor p* $^{*}$} \\
\cline { 3 - 5 } & & Mediana & \multicolumn{2}{|c|}{ IQR } & \\
\hline Caliche con gallinaza (30) & $29(96.6)$ & 37 & 28 & 48 & \multirow{2}{*}{0.013} \\
\hline Fértil con gallinaza (30) & $30(100)$ & 30 & 21 & 37 & \\
\hline Caliche sin gallinaza (30) & $24(80)$ & 37 & 27 & 80 & \\
\hline Fértil sin gallinaza (30) & $30(100)$ & 30 & 25 & 42 & \\
\hline
\end{tabular}

${ }^{£}$ Valor $\mathrm{p}$ de Prueba exacta de Fisher $=0.004$

*Prueba Log-rank

Según el sustrato elegido, como se muestra en la tabla 3, el fértil con y sin gallinaza compostada permitió la formación de raíces de todos los cladodios sembrados. Adicionalmente, ambos sustratos permitieron esta formación más rápido (mediana 30 días), pero el que tenía gallinaza compostada tuvo un rango intercuartílico de formación radicular más rápido y un poco más corto (21 a 37 días vs 25 a 42 días).

\section{REFERENCIAS BIBLIOGRAFICAS:}

- Castro J., Paredes C., Muñoz D. (Gerencia Regional Agraria La Libertad). (2009). Cultivo de Tuna. 5-13. Disponible en

http://www.agrolalibertad.gob.pe/sites/default/files/MANUAL\%20TECNICO\%20DE\%20TUN A.pdf.

- García E., Hernández I., Tarango L., Torres M., Becerra J., Pastor F., Martínez O., Valverde A., García J., Espinosa A., Cedillo I., Talavera D., Quero A., Amante A., Rossel D., Ortiz H., Tizcareño A. (2003). Características de la cadena agroalimentaria del Nopal Tunero e identificación de sus demandas tecnológicas. 5-7.

- Ríos J., Quintana V. Manejo general del cultivo del Nopal. (2004). 41 y 42. Tomado de http://www.elquiglobalenergy.com/english/datas/Manejo_general_cultivo_Nopal.pdf.

- Robles Fabián; MACÍAS Rubén; GRIJALVA Raúl. Tecnología de producción de nopal verdura para el noreste de Sonora. 2008, pp. 42 - 43. Tomado de:

http://biblioteca.inifap.gob.mx:8080/jspui/bitstream/handle/123456789/1615/ 